\title{
Development and Validation of Prognostic Nomogram for Elderly Breast Cancer: A Large-Cohort Retrospective Study
}

\section{Gangfeng Li \\ Dan Zhang}

Clinical Laboratory Center of Shaoxing People's Hospital (Shaoxing Hospital Zhejiang University School of Medcine), Shaoxing, Zhejiang, 312000, People's Republic of China
Correspondence: Dan Zhang

Clinical Laboratory Center of Shaoxing People's Hospital (Shaoxing Hospital Zhejiang University School of Medcine), Shaoxing, Zhejiang, 3I2000, People's Republic of China

Email 841726829@qq.com
Purpose: Our research aims to study the bone metastatic patterns and prognostic outcomes in elderly breast cancer (BC) and to develop elder-specific nomograms.

Methods: We downloaded the data of BC patients between 2010 and 2016 from the Surveillance, Epidemiology, and End Results database. The differences in clinical features and prognosis between young (age $<65)$ and elderly (age $\geq 65)$ BC patients were compared. The univariate and multivariate Cox analyses were used to determine the overall survival (OS)- and cancer-specific survival (CSS)-related variables and establish two nomograms of $\mathrm{BC}$ patients with bone metastasis $(\mathrm{BCBM})$. The receiver operating characteristic (ROC) curve with area under the curve (AUC), calibration curve, decision curve analysis (DCA), and Kaplan-Meier survival curve were selected to evaluate nomograms.

Results: A total of 230,177 BC patients were enrolled in our research, including 142,025 young and 88,152 elderly patients. The prognosis of elderly BCBM patients was significantly worse than young patients. Age, race, breast subtype, tumor size, tumor grade, brain metastasis, liver metastasis, surgery, and chemotherapy were independent prognostic variables for elderly BCBM patients, including OS and CSS. The AUC values at 12, 18, and 24 months were $0.750,0.751$, and 0.739 for OS nomogram and $0.759,0.762$, and 0.752 for CSS nomogram in the training cohort, which were higher than the AUC values of all single independent prognostic variables. The survival curve showed a distinct prognosis between low-, median- and high-risk groups $(p<0.001)$. Finally, calibration curves and DCA indicated that both nomograms have favorable performance.

Conclusion: Elderly and young patients presented with different bone metastatic frequencies, clinical features, and prognostic outcomes. Two elder-specific nomograms incorporating nine clinical variables were established and validated to be a valuable predictor for elderly BCBM patients.

Keywords: elder patients, breast cancer, bone metastasis, nomogram, overall survival, cancer-specific survival

\section{Introduction}

Breast cancer (BC) is the most common cancer and the second leading cause of death among women globally. ${ }^{1}$ With the rapid development of treatment methods such as surgery, chemotherapy, and immunotherapy, the treatment effectiveness of primary BC has been dramatically improved. However, distant metastases led to $80 \%$ of cancerassociated death, which gradually become one of the hot issues for oncologist. ${ }^{2}$

Bone metastasis (BM) is one of the most frequent metastatic patterns for $\mathrm{BC}$ patients, accounting for $41.4-65.9 \%$ of all metastatic cases. ${ }^{3,4}$ Recent progress in $\mathrm{BC}$ therapy has improved the patients' prognosis but conversely increased the 
BM risk. ${ }^{5}$ Due to non-response to chemotherapy, incomplete surgical resection, and resistance to checkpoint inhibitors, $\mathrm{BC}$ with $\mathrm{BM}$ (BCBM) is notoriously difficult to cure. The median survival for BCBM patients was only 19 months, and the 5-year survival rate was only $13 \%{ }^{6,7}$ Recently, several studies were conducted to study the prognostic factors for BCBM patients. Clinicopathological data, laboratory tests, and genomic data were confirmed as prognostic predictors for BCBM patients, ${ }^{8-16}$ and several studies aimed to develop prognostic nomograms for BCBM patients. ${ }^{6,16-19}$ However, elderly BC patients have their own unique characteristics, which suggested that we need to conduct a specific study for this special population. ${ }^{20-22}$

In this study, we obtained population-based data of BC patients to investigate the characteristics of elderly $\mathrm{BC}$ patients and aimed to establish two novel nomograms to predict the overall survival (OS) and cancer-specific survival (CSS) of elderly BCBM patients.

\section{Methods}

\section{Data and Cohort Definition}

The data of present study cohort was extracted from the Surveillance, Epidemiology, and End Results (SEER) database using the SEER*Stat software (version 8.3.6). Adult female patients ( $\geq 18$ years) diagnosed as BC between 2010 and 2016 were included. The information obtained in our study for each patient include age at diagnosis, race, histological type, tumor grade, T stage, N stage, surgery, radiotherapy, chemotherapy, distant metastasis (bone, liver, brain, and lung), tumor size, tumor subtype, marital status, cause of death, vital status, and survival time. Patients lacking one or more above characteristics data were excluded from this study. Meanwhile, patients with previous diagnosed tumor or survival time less than one month were excluded. All included patients were included and divided into young (age $<65$ ) and elderly groups (age $\geq 65$ ). The prognostic difference between young and elderly patients was compared with Kaplan-Meier (K-M) and the multivariate Cox regression models were used to adjust the potential confounding factors.

\section{Statistical Analysis}

All statistical analyses in the present study were performed in the $\mathrm{R}$ software (version 3.6.1). Two-sided $\mathrm{P}$ value $<0.05$ was considered as statistically significant. Categorical variables are reported as frequencies and proportions. Continuous variables are reported as means (standard deviation, SD) and medians (interquartile ranges, IQR). The Student's $t$-test and chi-square test were performed to identify the difference between clinicopathological data between young and elderly patients.

Furthermore, we focused on the elderly group to develop elder-specific nomograms. First, all elderly BCBM patients were randomly divided into training $(70 \%)$ and validation (30\%) cohorts. The nomograms were developed in the training cohort and validated in the validation cohort. The X-tile software was used to determine the optimal cutoff value of tumor size and age. The univariate and multivariate Cox analyses were performed to determine the independent OS- and CSS-related variables. Subsequently, two nomograms were developed based on the independent OS- and CSS-related variables, respectively. The time-dependent receiver operating characteristic (ROC) curve with area under the curve (AUC) was used to evaluate the discrimination of nomograms. The calibration curve and decision curve analysis (DCA) were used to show the calibration and clinical utilization of nomograms, respectively.

To confirm that our nomograms are robust tools for predicting the prognosis for elderly patients, more comprehensive analyses were performed. According to the total point of each patient, the optimal cutoff points were determined by the X-tile software and the K-M survival curve with a Log rank test was used to show the prognostic difference of different risk groups. In addition, to confirm that the predictive ability of our nomograms was better than single prognostic variables, the timedependent ROC curves of all independent prognostic variables were generated to compared with the nomogram.

\section{Results}

\section{Patient Characteristics}

Totally, 230,177 BC patients were enrolled, including 142,025 young and 88,152 elderly patients. The detailed information of included patients is shown in Table 1 . The median follow-up time was 40 (IQR: 23-60) months. A total of 23,852 patients dead during the follow-up period, and 14,295 patients dead due to the breast cancer. Compared with young BC patients, elderly patients tended to have a higher incidence of Luminal A subtype, higher incidence of the white race, higher rate of widowed status, higher rate of Lobular type, lower tumor grade, smaller tumor size, lower $\mathrm{T}$ and $\mathrm{N}$ stage, and lower incidence of bone, brain, liver, and lung metastases (Table 1). For the 
Table I Baseline Clinical Characteristics of Younger and Elderly Breast Cancer Patients

\begin{tabular}{|c|c|c|c|}
\hline Characteristics & $\begin{array}{c}\text { Age }<65 \\
(n=142,025)\end{array}$ & $\begin{array}{c}\text { Age } \geq 65 \\
(n=88,152)\end{array}$ & $P$ \\
\hline Molecular subtype & & & $<0.001$ \\
\hline $\mathrm{HR}+/ \mathrm{HER} 2-$ & 99,811 & 70,399 & \\
\hline $\mathrm{HR}+/ \mathrm{HER} 2+$ & 17,396 & 6851 & \\
\hline HR-/HER2+ & 7228 & 2787 & \\
\hline TNBC & 17590 & 8115 & \\
\hline Race & & & $<0.001$ \\
\hline Black & 17,207 & 7926 & \\
\hline White & 109,186 & 73,775 & \\
\hline Other & 15,632 & 6451 & \\
\hline Marital status & & & $<0.001$ \\
\hline Married & 91,520 & 43,386 & \\
\hline Unmarried/Domestic & 570 & 151 & \\
\hline \multicolumn{4}{|l|}{ Partner } \\
\hline Divorced & 16,087 & 9921 & \\
\hline Separated & 2049 & 495 & \\
\hline Widowed & 5215 & 25,407 & \\
\hline Single (never married) & 26,584 & 8792 & \\
\hline Histological type & & & $<0.001$ \\
\hline Ductal & 111,160 & 63,913 & \\
\hline Lobular & 10,983 & 9355 & \\
\hline Mixed ductal and lobular & 7989 & 5152 & \\
\hline Others & 11,893 & 9732 & \\
\hline Tumor grade & & & $<0.001$ \\
\hline 1 & 29515 & 24,146 & \\
\hline ॥ & 60061 & 41,410 & \\
\hline III & 52018 & 22,410 & \\
\hline IV & 431 & 186 & \\
\hline Tumor size, mm & $24.20 \pm 23.92$ & $21.39 \pm 20.98$ & $<0.001$ \\
\hline T stage & & & $<0.001$ \\
\hline $\mathrm{TI}$ & 80,248 & 56,053 & \\
\hline $\mathrm{T} 2$ & 47,158 & 24,885 & \\
\hline T3 & 10,202 & 4261 & \\
\hline $\mathrm{T} 4$ & 4417 & 2953 & \\
\hline $\mathrm{N}$ stage & & & $<0.001$ \\
\hline No & 90,550 & 65,852 & \\
\hline $\mathrm{NI}$ & 37,908 & 16,366 & \\
\hline N2 & 8513 & 3649 & \\
\hline N3 & 5054 & 2285 & \\
\hline Bone metastasis & 3275 & 1779 & $<0.001$ \\
\hline Brain metastasis & 331 & 147 & 0.001 \\
\hline Liver metastasis & 1379 & 556 & $<0.001$ \\
\hline Lung metastasis & $136 \mid$ & 965 & 0.001 \\
\hline Surgery performed & 135,529 & 83,121 & $<0.001$ \\
\hline Chemotherapy performed & 76,848 & 20,007 & $<0.001$ \\
\hline Radiation therapy performed & 81,923 & 46,263 & $<0.001$ \\
\hline
\end{tabular}

treatment, the rates of surgery, chemotherapy, and radiotherapy in the elderly group were all significantly lower than young patients (Table 1). Generally, compared with young patients, elderly patients tended to have favorable tumor status but poor treatment status.
Among 230,177 BC patients, 5054 patients (2.2\%) were diagnosed with $\mathrm{BM}$ at presentation. The incidence of BM was higher than other distant sites, including lung $(1.0 \%)$, liver $(0.8 \%)$, and brain $(0.2 \%)$. The incidence of BM for elderly patients was significantly lower than young patients $(2.0 \%$ verse $2.3 \%, p<0.002)$ (Table 1$)$. To further validate this finding, the multivariate logistic analysis was performed to adjust for confounding variables including race, marital status, histological type, tumor grade, $\mathrm{T}$ stage, $\mathrm{N}$ stage, tumor size, and molecular subtype. The results showed that the young group tended to have more BM [Odds ratio $(\mathrm{OR})=1.078,95 \%$ confidence interval $(\mathrm{CI})$ $1.008-1.154, p=0.029]$. Similar with the total cohort, there were statistically significant differences in demographic information, primary tumor status, treatment, and distant metastasis between young and elderly BCBM patients (Table 2). However, there was not significant difference in tumor size between young and elderly BCBM patients (Table 2).

\section{Prognostic Difference Between Young and Elderly BCBM Patients}

Two K-M survival curves are shown in Figure 1, which show that the OS and CSS of young and elderly BCBM patients. Log rank tests indicated that elderly BCBM patients have a worse prognosis than young patients (Figure 1A and B). After adjusting for confounding variables, the multivariate analysis confirmed that age was an independent prognostic factor for OS [Hazard ratio (HR) 1.306, 95\% CI 1.199-1.423, $p<0.001$ ] (Figure 1A) and CSS [Hazard ratio (HR) 1.231, 95\% CI 1.125-1.347, $\mathrm{p}<0.001$ ] (Figure 1B). We further investigated the impact of age on the prognosis in different molecular subtypes. The elderly cohort showed significantly worse OS and CSS than the young cohort in HR+/HER2-, HR+/HER2+, and HR-/HER2+ subtypes (Figure $1 \mathrm{C}-\mathrm{G}$ and I). The prognosis of the elderly TNBC cohort was worse than the young TNBC cohort, but there was no significance (Figure 1H and J). Thus, we speculated that it may be attributed to the extremely poor prognosis of TBNC patients.

\section{Prognostic Factors for Elderly BCBM}

The optimal cutoff values for age and tumor size were 80 years and $72 \mathrm{~mm}$, respectively. Patients aged 65-79 years were included in the relatively young group, while patients aged $\geq 80$ years were included in the relatively old group. Meanwhile, patients with tumor size of 1-72 mm were included in the small tumor group, while patients with 
Table 2 Baseline Clinical Characteristics of Younger and Elderly BCBM

\begin{tabular}{|c|c|c|c|}
\hline Characteristics & $\begin{array}{c}\text { Age }<65 \\
(n=3275)\end{array}$ & $\begin{array}{c}\text { Age } \geq 65 \\
(n=1779)\end{array}$ & $P$ \\
\hline Molecular subtype & & & $<0.001$ \\
\hline HR+/HER2- & 2076 & 1319 & \\
\hline HR+/HER2+ & 633 & 225 & \\
\hline HR-/HER2+ & 244 & 75 & \\
\hline TNBC & 322 & 160 & \\
\hline Race & & & $<0.001$ \\
\hline Black & 584 & $2,232,569$ & \\
\hline White & 2397 & 1464 & \\
\hline Other & 294 & 92 & \\
\hline Marital status & & & $<0.001$ \\
\hline Married & 1723 & 731 & \\
\hline Unmarried/Domestic & 14 & 2 & \\
\hline Partner & & & \\
\hline Divorced & 389 & 242 & \\
\hline Separated & 72 & 14 & \\
\hline Widowed & 159 & 541 & \\
\hline Single (never married) & 918 & 249 & \\
\hline Histological type & & & $<0.001$ \\
\hline Ductal & 2569 & 1280 & \\
\hline Lobular & 299 & 260 & \\
\hline Mixed ductal and lobular & 162 & 113 & \\
\hline Others & 245 & 126 & \\
\hline Tumor grade & & & $<0.001$ \\
\hline 1 & 255 & 200 & \\
\hline II & 1480 & 910 & \\
\hline III & 1526 & 665 & \\
\hline IV & 14 & 4 & \\
\hline Tumor size, mm & $50.90 \pm 42.79$ & $49.32 \pm 41.90$ & 0.208 \\
\hline T stage & & & 0.007 \\
\hline $\mathrm{Tl}$ & 399 & 261 & \\
\hline $\mathrm{T} 2$ & 1253 & 636 & \\
\hline T3 & 681 & 331 & \\
\hline $\mathrm{T} 4$ & 942 & 551 & \\
\hline $\mathrm{N}$ stage & & & $<0.001$ \\
\hline No & 637 & 479 & \\
\hline $\mathrm{NI}$ & 1611 & 821 & \\
\hline N2 & 480 & 219 & \\
\hline N3 & 547 & 260 & \\
\hline Brain metastasis & 212 & 82 & 0.007 \\
\hline Liver metastasis & 795 & 301 & $<0.001$ \\
\hline Lung metastasis & 734 & 455 & 0.011 \\
\hline Surgery performed & 1293 & 586 & $<0.001$ \\
\hline Chemotherapy performed & 2224 & 687 & $<0.001$ \\
\hline $\begin{array}{l}\text { Radiation therapy } \\
\text { performed }\end{array}$ & 1493 & 647 & $<0.001$ \\
\hline
\end{tabular}

Abbreviation: $\mathrm{BCBM}$, breast cancer with bone metastasis.

tumor size $\geq 73 \mathrm{~mm}$ were included in the large tumor group. In the univariate Cox analysis, twelve variables were confirmed as OS-related factors, and eleven variables were confirmed as CSS-related factors (Figure S1). Furthermore, multivariate Cox analysis indicated that age, race, grade, surgery, chemotherapy, brain metastasis, liver metastasis, tumor size, and breast subtype were independent OS- and CSS-related factors (Figures 2 and $\underline{\mathrm{S}}$ ).

\section{Elderly-Specific Nomograms Construction}

Two nomograms integrating nine independent prognostic factors were constructed (Figure 3A and B). The scores assigned to each variable can be viewed in detail in Table 3. By adding individual score of those nine variables, doctors could obtain the total score and determine a specific probability of 12,18 , and 24 months survival. The AUC values of OS nomogram in the training cohort were $0.750,0.751$, and 0.739 at 12,18 , and 24 months, respectively (Figure 4A). Additionally, the AUC values of the CSS nomogram in the training cohort were 0.759 , 0.762 , and 0.752 at $12-, 18$, and 24 months, respectively (Figure 4D). The calibration curves of nomograms in the training cohort indicated favorable consistencies between the predicted and actual prognosis (Figure 5A-F). The DCA curves for the training cohort at 12, 18, and 24 months were also generated and shown in Figure 6A-F. The nomogram showed great positive net benefits across wide ranges of death risk in the training cohort, indicating its favorable clinical utility in predicting 12,18 , and 24 months OS and CSS.

\section{Validation of Nomograms}

Totally, 532 patients were enrolled in the validation cohort. In the validation cohort, the AUC values of the OS nomogram were $0.731,0.721$, and 0.699 at 12,18 , and 24 months (Figure 4G). Similarly, the AUC values of the CSS nomogram were also satisfactory, which were higher than 0.710 at three time points (Figure 4J). The calibration curves and DCA results of nomograms in the validation cohort also showed favorable performance (Figures $5 \mathrm{G}-\mathrm{L}$ and $6 \mathrm{G}-\mathrm{L}$ ).

\section{Risk Stratification Model for Elderly BCBM Patients}

According to each patient's score from the nomogram, the cutoff values were determined in the X-tile software. For the OS nomogram, 255 and 309 were two cut points, and all patients were stratified into three groups: low-risk group $(\mathrm{n}=433,34.7 \%$; total score $<255)$, middle-risk group ( $\mathrm{n}=672,53.9 \%$; total score 255-309), and high-risk group $(\mathrm{n}=142,11.4 \%$; total score $>309)$. Similarly, 263 and 313 were identified as two cut points for CSS 

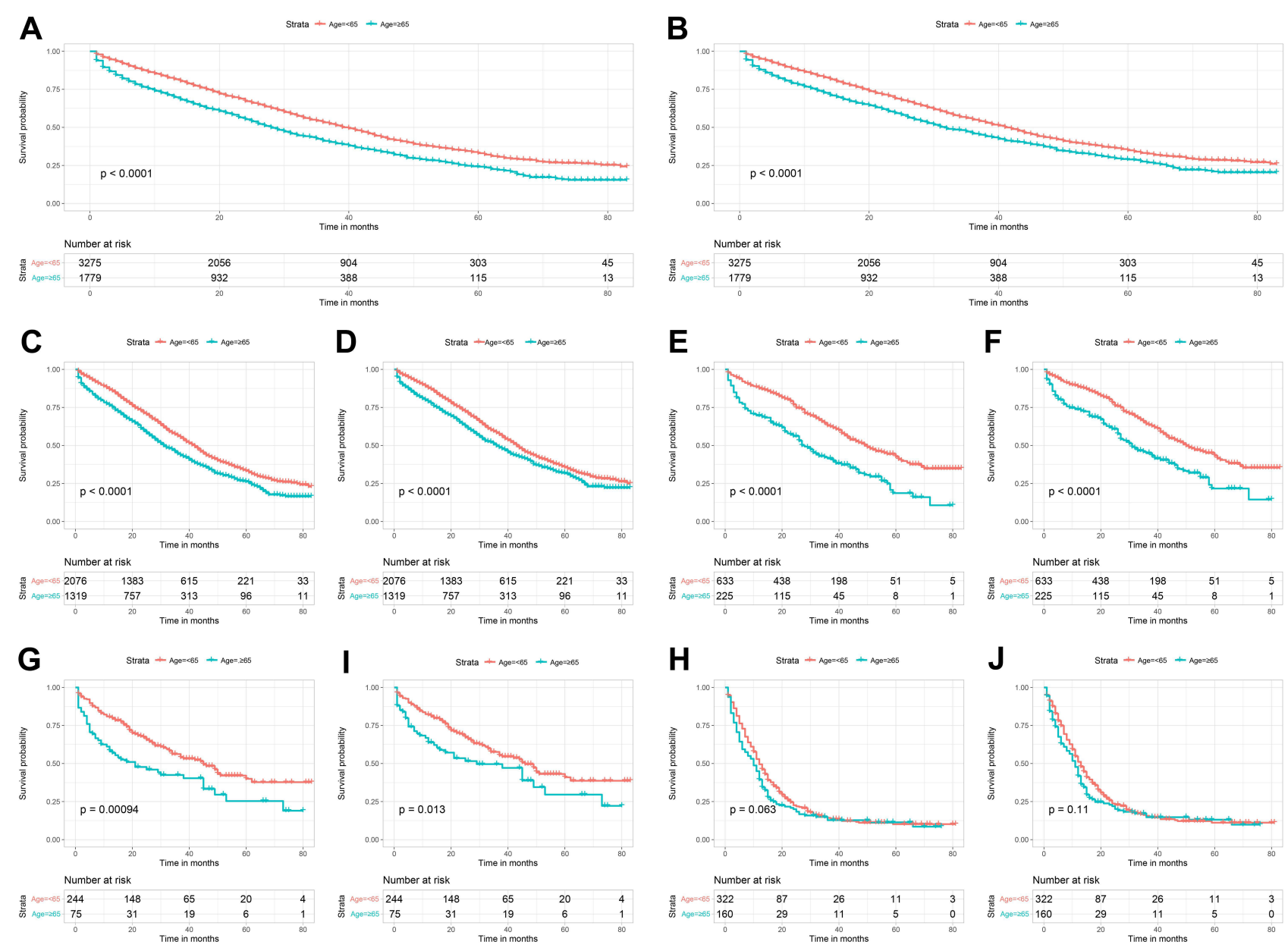

Figure I Survival curves showed the distinct prognosis between young and elderly breast cancer patients with bone metastasis. (A) Overall survival in all breast cancer patients with bone metastasis. (B) Cancer-specific survival in all breast cancer patients with bone metastasis. (C) Overall survival in total Luminal A (HR+/HER2-) patients with bone metastasis. (D) Overall survival in total Luminal B (HR+/HER2+) patients with bone metastasis. (E) Cancer-specific survival in total Luminal A (HR+/HER2-) patients with bone metastasis. (F) Cancer-specific survival in total Luminal B (HR+/HER2+) patients with bone metastasis. (G) Overall survival in HER2 enriched (HR-/HER2 + ) cohort. (H) Overall survival in triple-negative (HR-/HER2-) patients with bone metastasis. (I) Cancer-specific survival in HER2 enriched (HR-/HER2+) patients with bone metastasis. (J) Cancer-specific survival in triple-negative (HR-/HER2-) patients with bone metastasis.

Abbreviations: HR, hormone receptor; HER2, human epidermal growth factor receptor 2.

signature. The prognosis was significantly different between three risk groups (Figure 4B, C, E and F). For the validation cohort, the cut points in the training cohort were used. K-M survival curves also indicated a significantly distinct outcome between three risk groups (Figure 4H, I, K and L).

\section{Subgroup Analysis of Nomograms}

According to the ROC analysis and K-M survival analysis, we can find that both OS nomogram and CSS nomogram can effectively predict the prognosis of elderly BCBM patients. However, whether these two nomograms perform well in subgroups is unclear. Therefore, we further performed the subgroup analysis to study the prognostic value of nomograms in the different age and grade groups. In the training cohort, both nomograms showed satisfactory prognostic differentiation ability in four subgroups (Figure 7A-D). In all subgroups, patients at high risk had a worse prognosis than those at middle risk, and patients in the low-risk group had the best prognosis (Figure 7A-D). The above results were also confirmed in the validation cohort (Figure 7E-H).

\section{Comparison of Predictive Accuracy Between the Nomogram and a Single Independent Factor}

As shown in Figure 2, nine independent prognostic variables were confirmed. The ROC curves of these variables are shown in Figure 8. Generally, the AUC values of all independent prognostic variables were higher than 0.500 . 


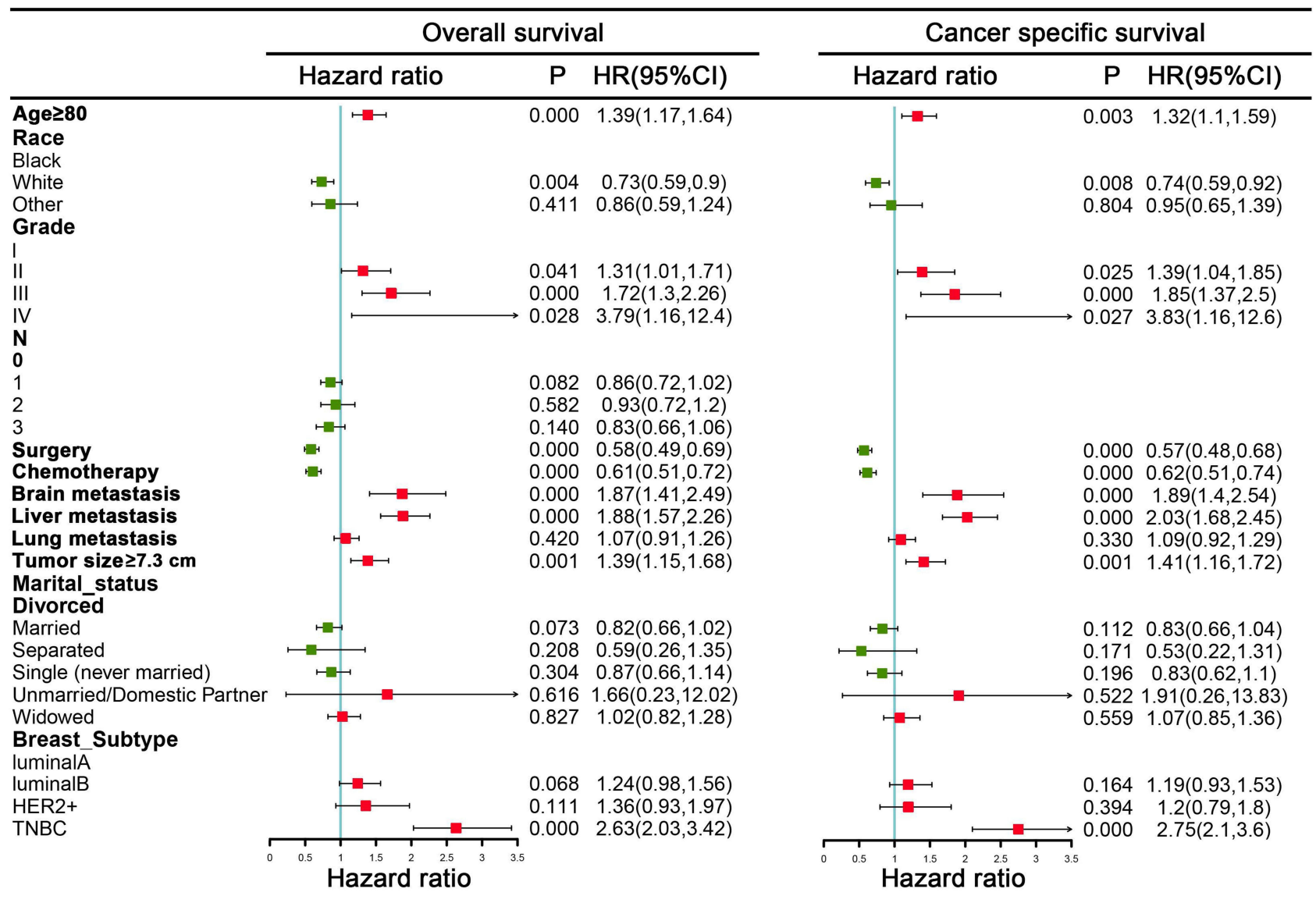

Figure 2 The forest plot showed the results of multivariate Cox analyses for elderly breast cancer patients with bone metastasis. Abbreviations: HER2, human epidermal growth factor receptor 2; TNBC, triple negative breast cancer; HR, hazard ratio.

In the training cohort, tumor grade and tumor subtype were the top two strongest prognostic variables (Figure 8A-F). In the validation cohort, the AUC values of liver metastasis were always higher than other indicators at 12, 18, and 24 months (Figure 8G-L). However, the AUC values of all single independent factors were lower than the AUC values of nomograms (Figure 8A-L).

\section{Discussion}

Bone is one of the most common metastatic sites in $\mathrm{BC}$ patients. The incidence, risk factors, and prognostic factors have been reported in recent studies. The nomograms for predicting the prognosis for BCBM patients were developed by Wang et al. ${ }^{16}$ However, it is still controversial whether elderly BCBM patients were distinct from young patients. In this study, our results suggested that the incidence of $\mathrm{BM}$ in the elderly $\mathrm{BC}$ patients is lower but the prognosis is poor. Two nomograms incorporating nine clinicopathological variables were conducted and validated. The further comprehensive evaluation confirmed that our elderly specific nomograms have favorable discrimination, calibration, and clinical utilization. Finally, two risk stratification models were developed according to each patient's total score from the nomogram.

To our knowledge, it is the first study to establish prognostic models for elderly BCBM patients. Compared with previous nomograms, our nomograms have several advantages. First, compared with the nomograms developed by Wang, our nomograms were elderly-specific tools. Patients with $\mathrm{BC}$ in the elderly and young have distinct molecular characteristics and need different clinical management strategies. ${ }^{20-22}$ The present results also confirmed that are many differences between younger and older $\mathrm{BC}$ patients and our elder-specific nomograms were better predictors for elder BCBM patients. Secondly, BCBM patients older than 80 were included in our research, which were not included in Wang's study. ${ }^{16}$ Octogenarian $\mathrm{BC}$ patients have a poor prognosis, but risk stratification and the subgroup of women age $\geq 80$ years may benefit from more aggressive treatment. ${ }^{23,24}$ Finally, several novel tools and methods were used in the present study. The X-tile, a new bioinformatics tool for biomarker 

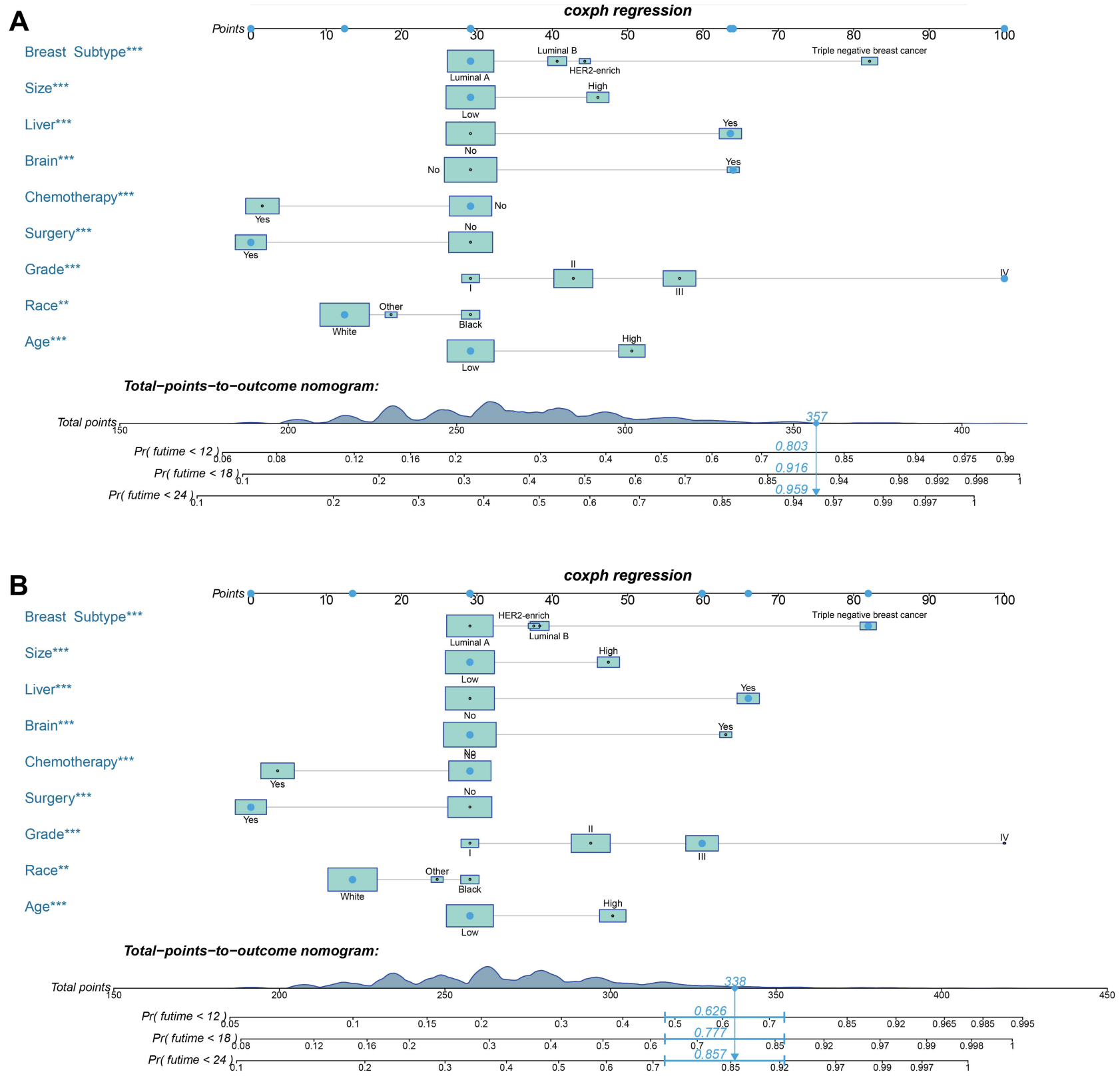

Figure 3 Two nomograms were generated for predicting OS and CSS for elderly BCBM patients, respectively. (A) Nomogram incorporating nine clinicopathological variables for predicting the OS of elderly BCBM patients; (B) nomogram incorporating nine clinicopathological variables for predicting the CSS of elderly BCBM patients. Abbreviations: OS, overall survival; CSS, cancer-specific survival; BCBM, breast cancer with bone metastasis.

assessment and outcome-based cut-point optimization, was selected to determine the optimal cutoff value of age, tumor size, and risk score. ${ }^{25}$ Based on the X-tile and the score of each patient, two risk stratification models were developed and K-M survival showed a distinct prognosis between three groups.

Age was determined as an independent prognostic factor for elderly BCBM patients. Although they were all older patients, we found that there was an age-related survival among them and patients with age $\geq 80$ have poorer OS and CSS. BC patients in the 80 and over age group were commonly diagnosed with advanced disease compared to younger patients who were diagnosed more commonly with early disease. ${ }^{26}$ Another novel reported finding in the $\mathrm{BC}$ patients $\geq 80$ years is that those patients discontinued the prescribed therapy earlier and more frequently than younger patients. ${ }^{27,28}$ The comprehensive influence of factors may result in the worse prognosis of octogenarian patients. Another prognostic demographic variable is race, which has been widely reported in 
Table 3 Point Assignment for Specific Categories of the Variables Included in the Nomograms

\begin{tabular}{|c|c|c|}
\hline Variable & OS Nomogram & CSS Nomogram \\
\hline \multicolumn{3}{|l|}{ Molecular subtype } \\
\hline HR+/HER2- & 29 & 29 \\
\hline HR+/HER2+ & 41 & 38 \\
\hline HR-/HER2+ & 44 & 38 \\
\hline TNBC & 82 & 82 \\
\hline \multicolumn{3}{|l|}{ Age, year } \\
\hline $65-79$ & 29 & 29 \\
\hline$\geq 80$ & 51 & 48 \\
\hline \multicolumn{3}{|l|}{ Race } \\
\hline Black & 29 & 29 \\
\hline White & 12 & 13 \\
\hline Other & 19 & 25 \\
\hline \multicolumn{3}{|l|}{ Tumor grade } \\
\hline $\mathrm{I}$ & 29 & 29 \\
\hline II & 43 & 45 \\
\hline III & 57 & 60 \\
\hline IV & 100 & 100 \\
\hline \multicolumn{3}{|l|}{ Tumor size, mm } \\
\hline I-72 & 29 & 29 \\
\hline$\geq 73$ & 46 & 47 \\
\hline \multicolumn{3}{|l|}{ Brain metastasis } \\
\hline No & 29 & 29 \\
\hline Yes & 64 & 63 \\
\hline \multicolumn{3}{|l|}{ Liver metastasis } \\
\hline No & 29 & 29 \\
\hline Yes & 64 & 66 \\
\hline \multicolumn{3}{|l|}{ Surgery } \\
\hline No & 29 & 29 \\
\hline Yes & 0 & 0 \\
\hline \multicolumn{3}{|l|}{ Chemotherapy } \\
\hline No & 29 & 29 \\
\hline Yes & 2 & 4 \\
\hline
\end{tabular}

previous studies. ${ }^{24,29-31}$ However, the race has a different effect on prognosis in different $\mathrm{BC}$ patients. In our research, African American patients have the worst prognosis, which maybe because they are usually diagnosed at an advanced stage.

For tumorous factors, tumor grade is the most important prognostic factor in our research. The higher the tumor grade, the worse the prognosis of the patients, which was consistent with the previous studies. ${ }^{16,24}$ Multiorgan and multiple BM were confirmed as risk factors for BCBM patients. ${ }^{10,16}$ In the present study, we precisely determine that liver and brain metastases, but not lung, were independent prognostic factors of elderly BCBM patients. Previous studies focused on BCBM patients of all ages confirmed that lung metastases was an independent risk factors. ${ }^{32,33}$ Combined with the results of this study, we found that lung metastasis appears to be an age-specific prognostic factor for BCBM patients. However, the mechanism of this results needs to be further studied and whether this phenomenon also exists in other tumors deserves further study. Tumor size was another prognostic factor for elderly BCBM patients. Using $\mathrm{X}$-tile software to convert continuous variables into categorical variables has been widely used in clinical cancer research. ${ }^{34,35}$ We determine $72 \mathrm{~mm}$ as the cutoff value of tumor size, which was proved to be a good way to stratify the prognosis of patients.

In our research, both surgery and chemotherapy were determined as protective factors. In recent years, several studies indicated that local surgery could achieve improvement in the prognosis of metastatic BC. ${ }^{36-40}$ Xiong et al reported that patients with $\mathrm{BM}$ alone (and primary tumor $\leq 5 \mathrm{~cm}$ ) can benefit from surgery. ${ }^{39}$ Consistently, a large cohort retrospective study indicated that local surgery had significantly improved OS in BCBM patients. ${ }^{40}$ However, one study has put forward a different conclusion that the resection of the primary tumor has no significant improvement in the prognosis of BCBM. ${ }^{41}$ Therefore, prospective and randomized controlled studies are needed to further study this scientific question. Chemotherapy is the fundamental treatment for BCBM patients and new chemotherapeutic agents such as etirinotecan and nab-paclitaxel have been established in recent years. ${ }^{42}$ However, the most effective regimen in the treatment of $\mathrm{BM}$ has not been defined. ${ }^{43}$ Treatments developed in recent years, such as cytokine therapy, bone-modifying agents, and smallmolecule inhibitor, are expected to become effective treatments for BCBM patients.

There are several limitations to our research. First, it is a retrospective study based on the SEER database. Some important prognostic data, such the detailed information about treatment and comorbidities of each patient were not available. Secondly, the old age was defined as being 65 or older in the present study. However, both in this study and in previous studies, patients $\geq 80 / 70$ years old appeared to have a poorer prognosis. ${ }^{4,24,44}$ It is not clear whether special studies are necessary for this super-aged group. Finally, although the prognostic nomograms and risk stratification models have been established for BCBM patients, the appropriate management for patients in each group remains unclear. 
A

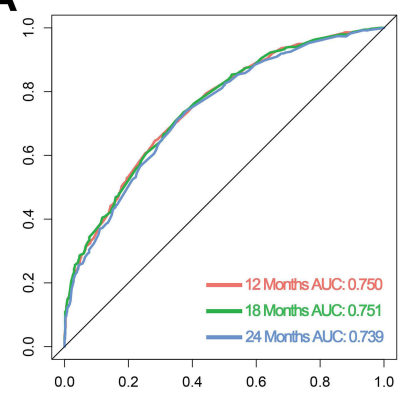

D

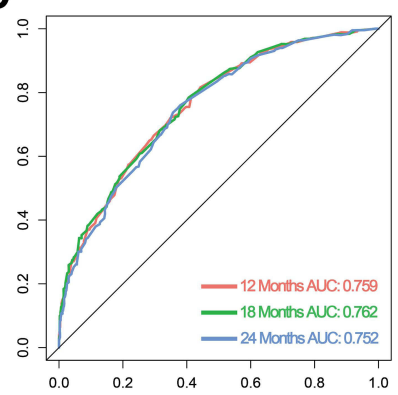

G

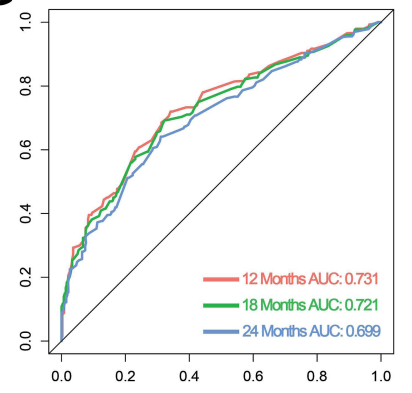

J

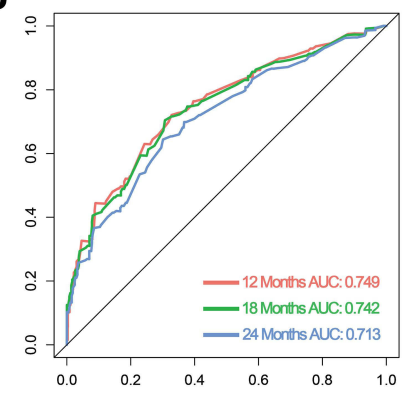

B
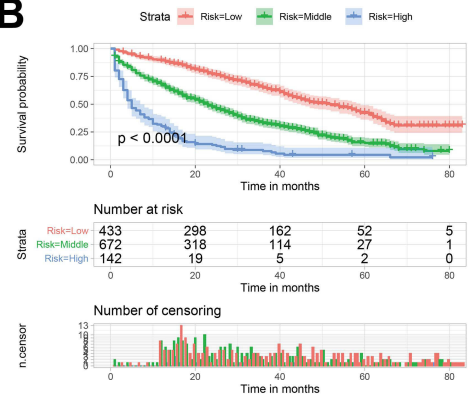

E

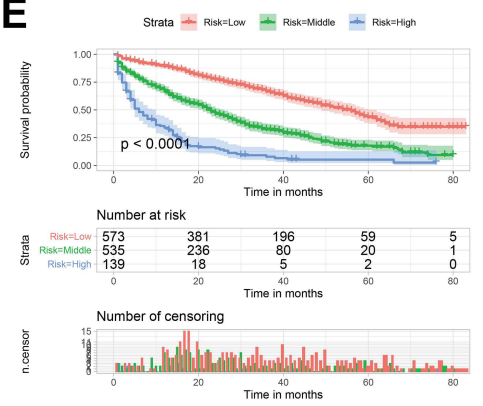

H

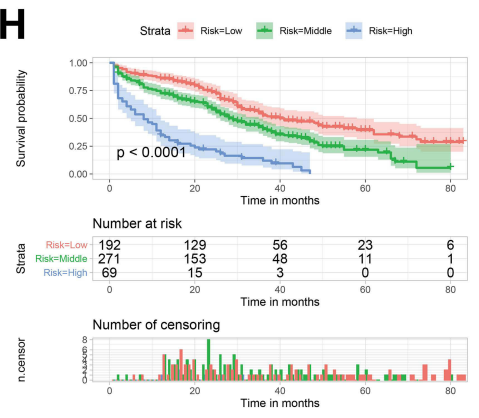

K

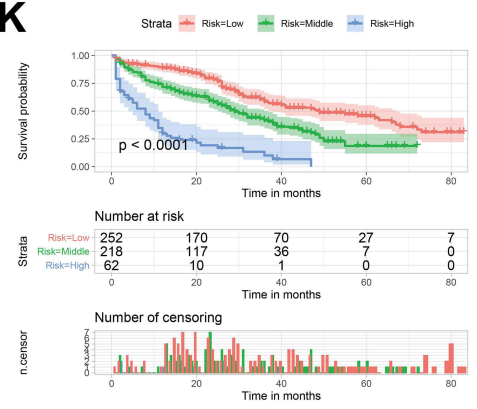

C

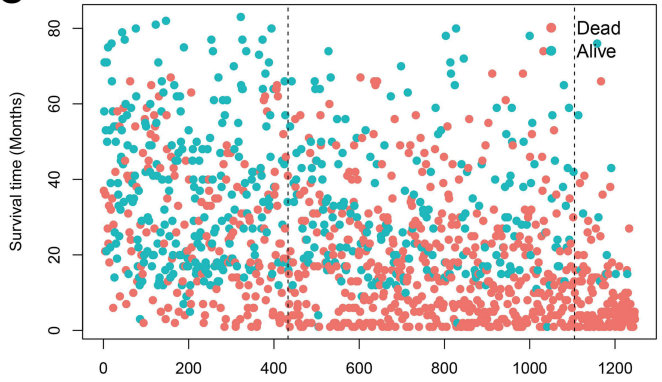

F

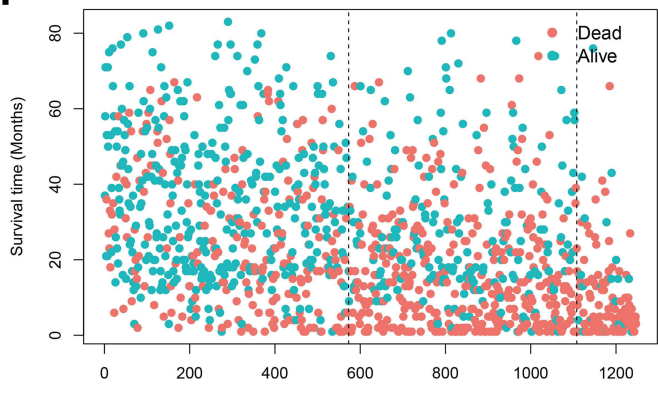

Patients (increasing risk socre)

I

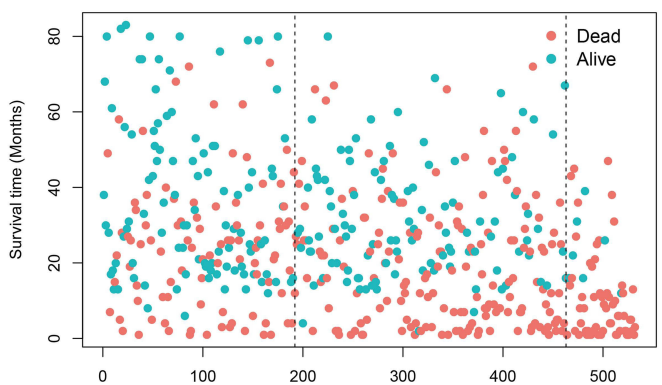

$\mathbf{L}$

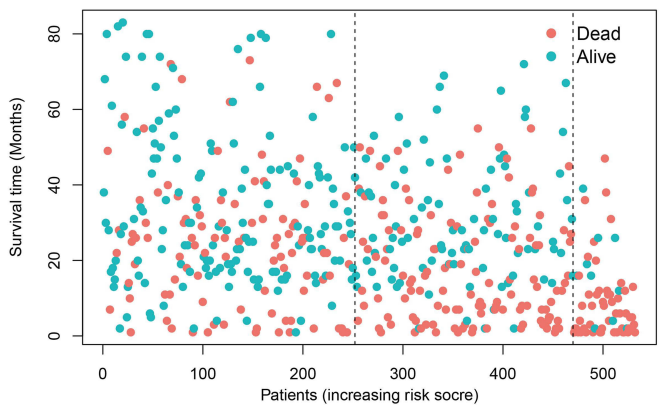

Figure 4 The ROC curve, survival curve, and survival status based on nomograms in both training and validation cohorts. (A, D, G and J) I2, I8, and 24 months ROC curves of OS ( $\mathbf{A}$ and $\mathbf{G})$ and CSS (D and $\mathbf{J})$ nomograms in the training ( $\mathbf{A}$ and $\mathbf{D})$ and validation $(\mathbf{G}$ and $\mathbf{J})$ cohorts; (B, E, $\mathbf{H}$ and $\mathbf{K})$ survival curves showed the distinct OS $(\mathbf{B}$ and $\mathbf{H})$ and CSS $(\mathbf{E}$ and $\mathbf{K})$ between three risk groups in the training $(\mathbf{B}$ and $\mathbf{E})$ and validation $(\mathbf{H}$ and $\mathbf{K})$ cohorts; $(\mathbf{C}, \mathbf{F}, \mathbf{I}$ and $\mathbf{L})$ survival status plots show the OS (C and $\mathbf{I})$ and CSS (F and $\mathbf{L})$ status in the training $(\mathbf{C}$ and $\mathbf{F})$ and validation ( $\mathbf{I}$ and $\mathbf{L})$ cohorts.

Abbreviations: ROC, receiver operating characteristic; OS, overall survival; CSS, cancer-specific survival; AUC, area under the curve.

\section{Conclusion}

In summary, this study found that elderly BC patients with a lower incidence of BM but with poor prognosis. Nine clinicopathological variables were significantly related to the prognosis of elderly BCBM. Two simple nomograms based on these variables had favorable discrimination, calibration, and clinical utility. It could be a useful tool for the patient's consultation and doctor's evaluation. 
A

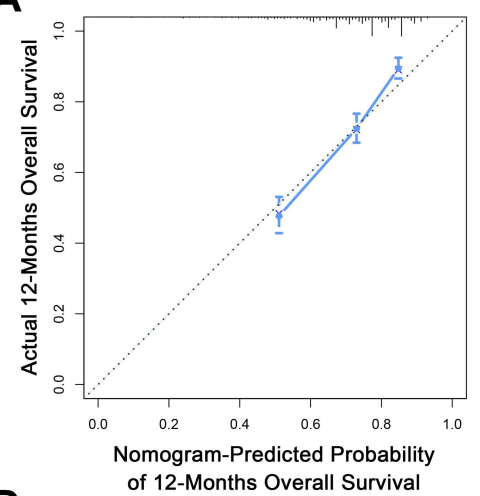

D

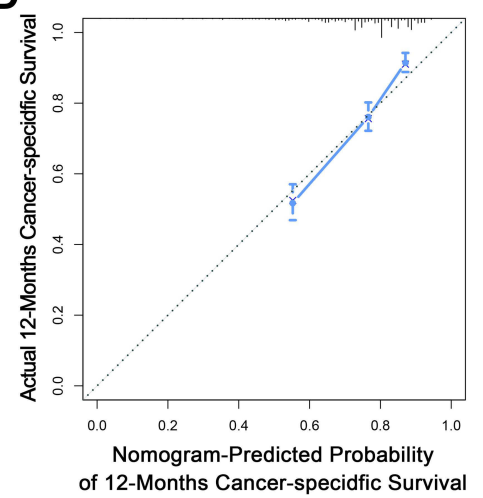

G

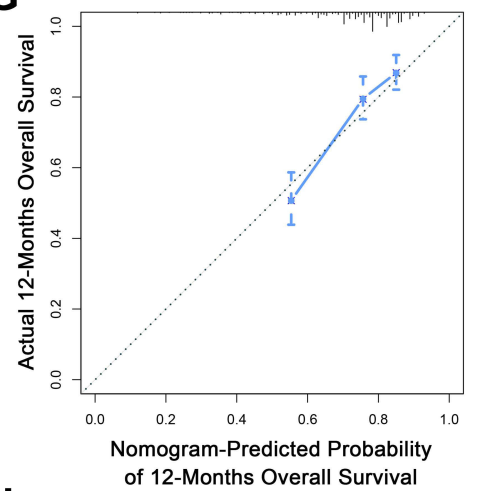

J

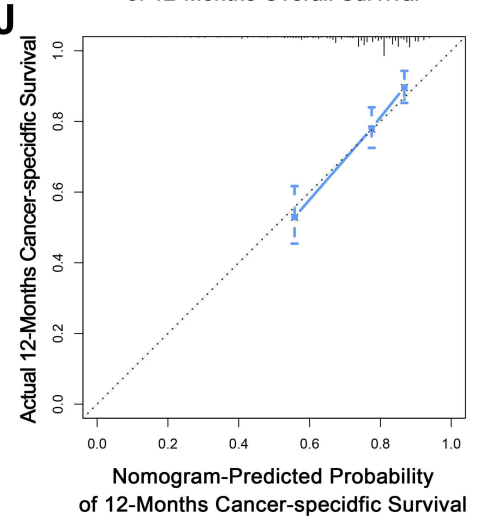

B

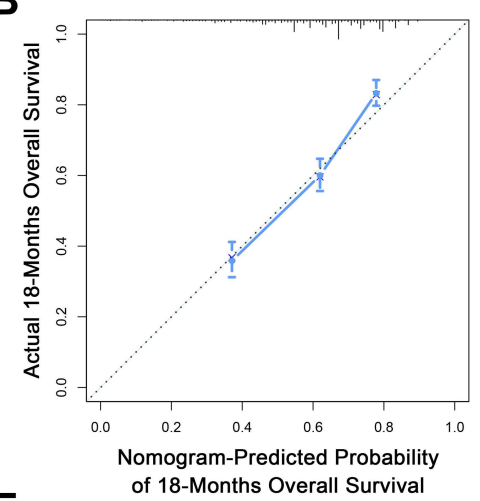

E

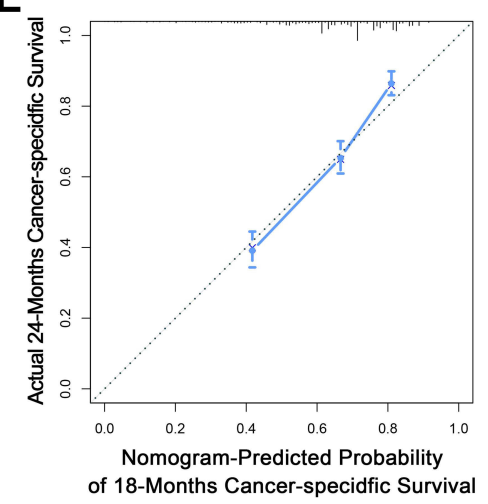

H

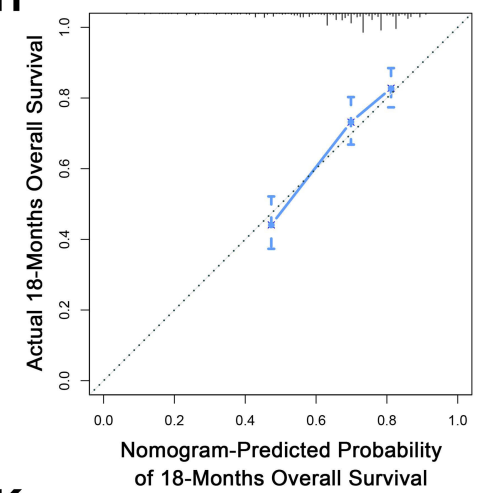

K

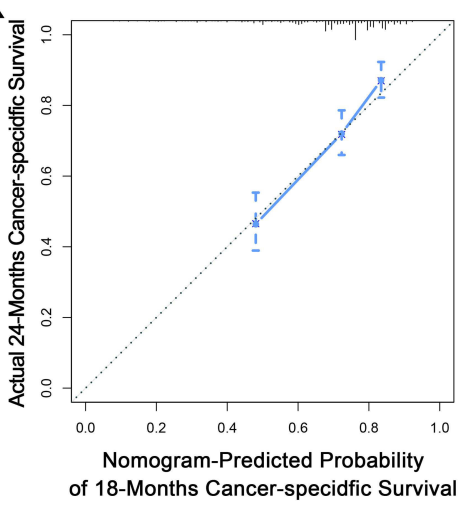

C

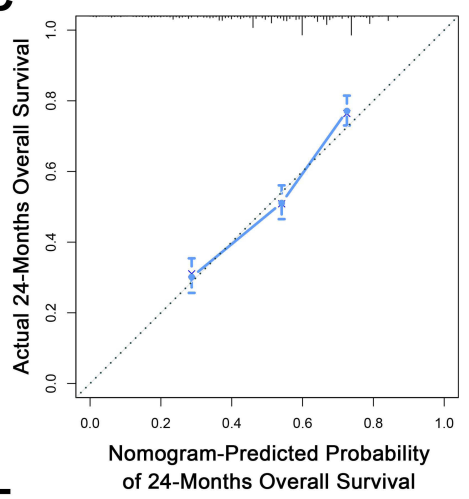

F

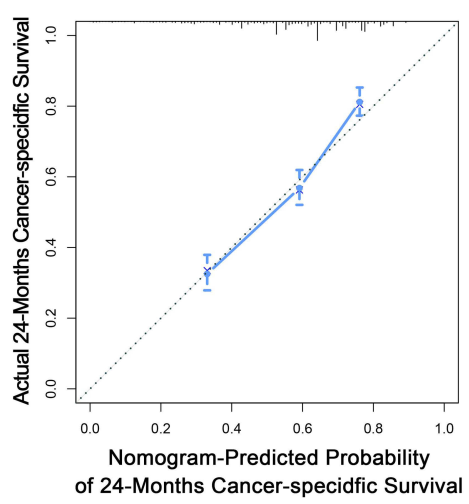

I

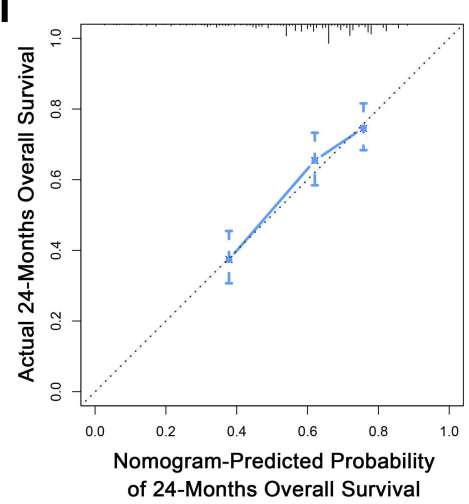

$\mathbf{L}$

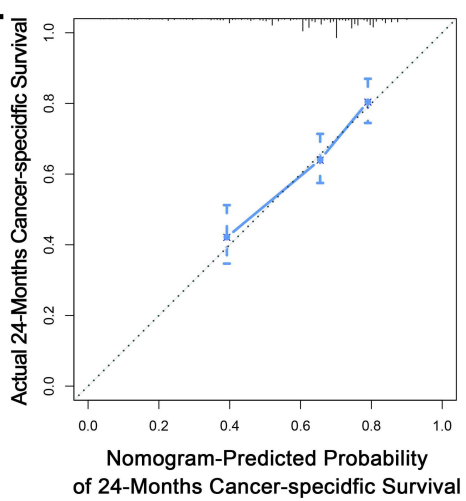

Figure 5 Calibration curves showed the calibration of OS and CSS nomograms in the training and validation cohorts. The calibration curves for OS (A-C) and CSS (D-F) nomograms in the training cohort. The calibration curves for OS (G-I) and CSS (J-L) nomograms in the validation cohort.

Abbreviations: OS, overall survival; CSS, cancer-specific survival. 

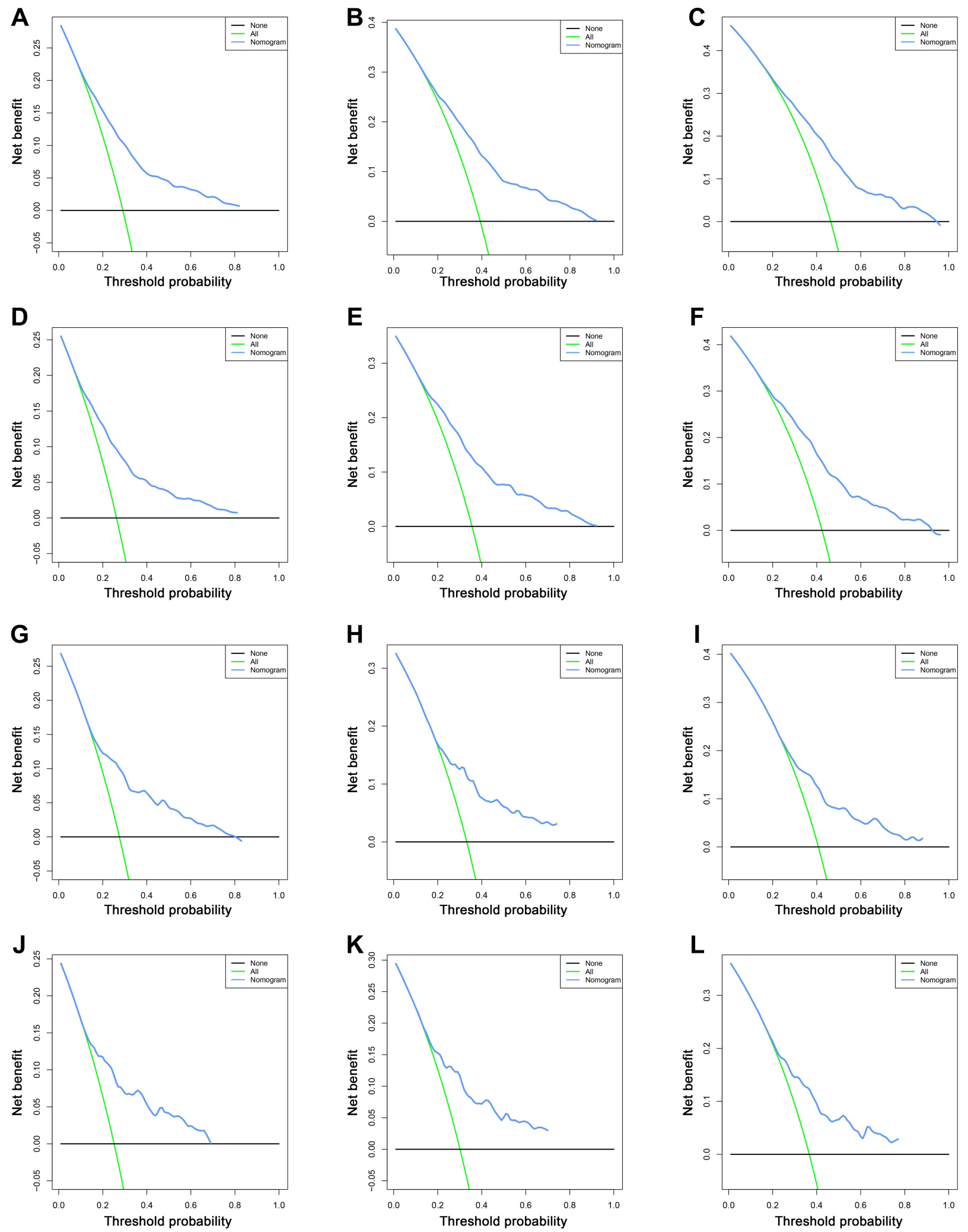

Figure 6 Decision curve analyses showed the calibration of OS and CSS nomograms in the training and validation cohorts. The decision curve analyses for OS (A-C) and CSS (D-F) nomograms in the training cohort. The decision curve analyses for OS (G-I) and CSS (J-L) nomograms in the validation cohort. Abbreviations: OS, overall survival; CSS, cancer-specific survival. 

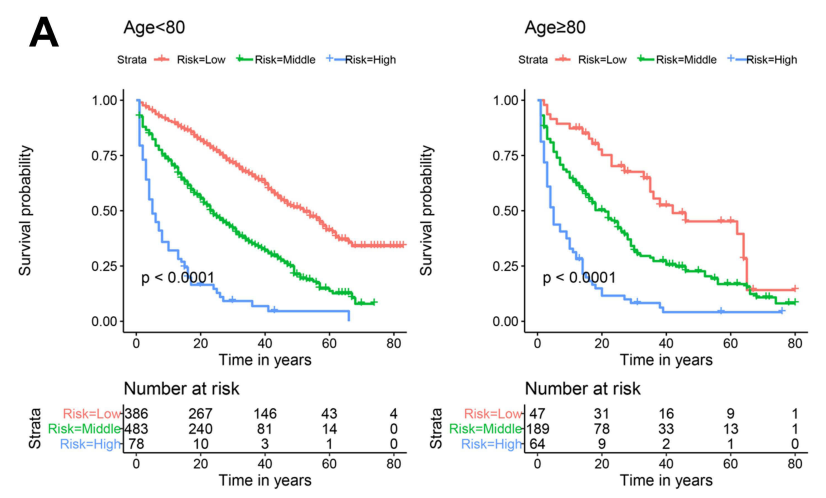

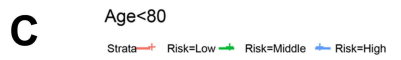

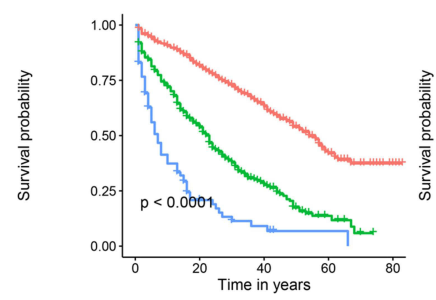

Number at risk

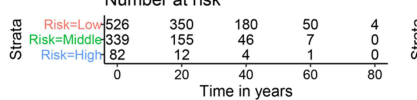

4
0
0
0
30

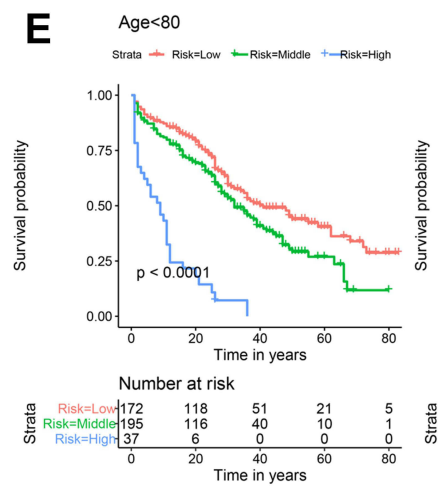

\section{G}
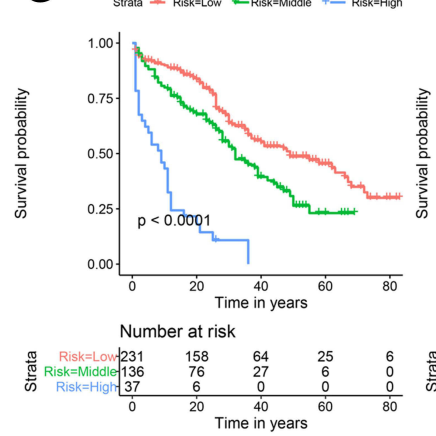

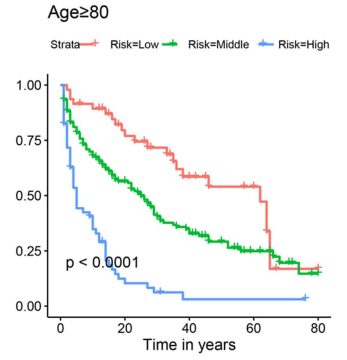

Number at risk

Age $\geq 80$
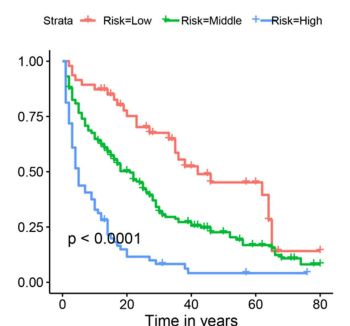

Number at risk
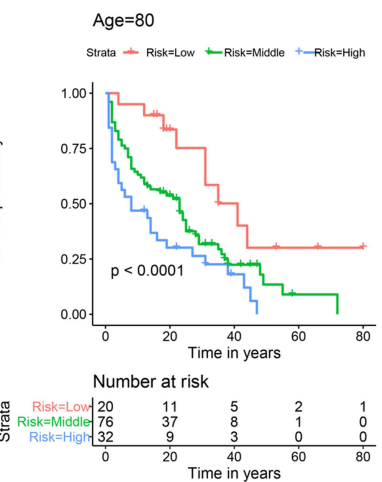

Age $\geq 80$

Strata + Risk=Low - Risk=Middle +- Risk=High

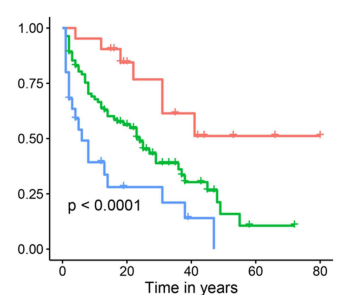

Number at risk
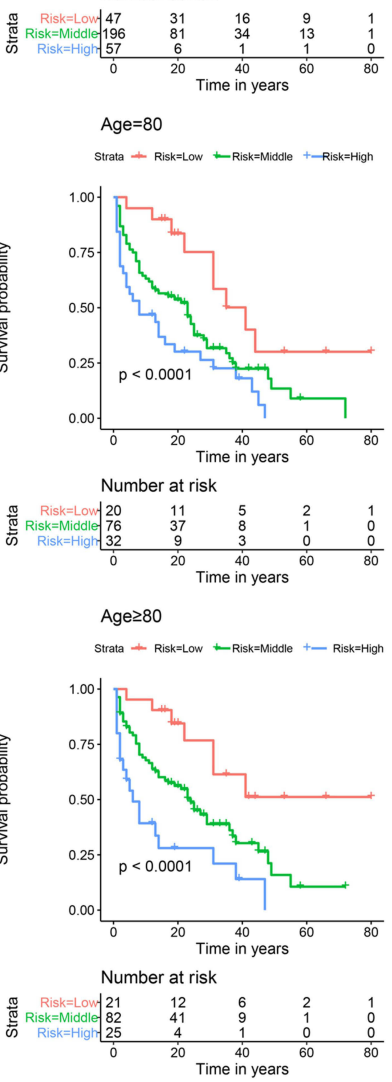
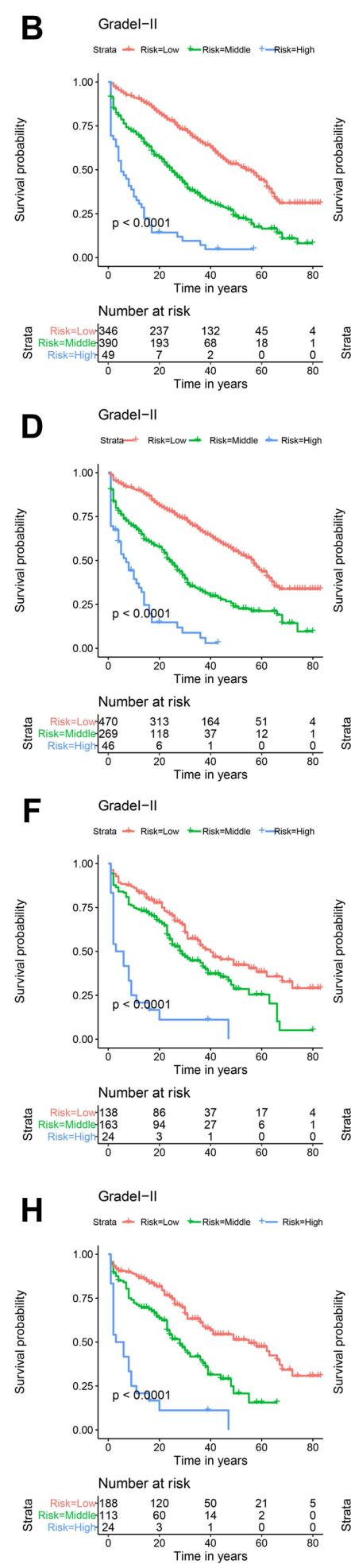

GradelII-IV

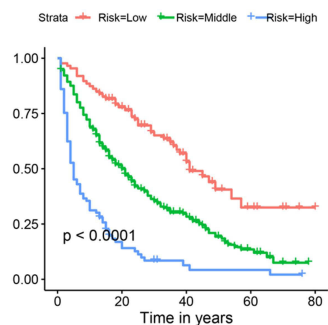

Number at risk

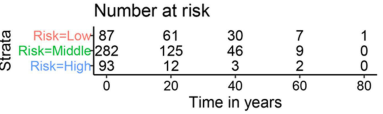

GradellI-IV
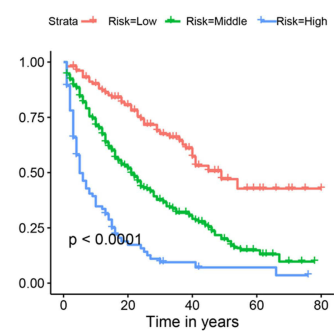

Number at risk
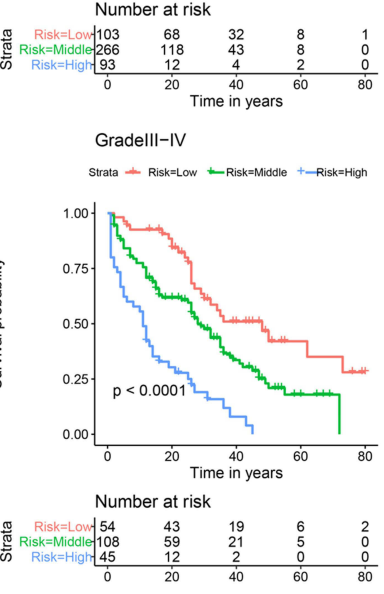

GradellI-IV

Strata - Risk=Low - Risk=Middle + - Risk=High

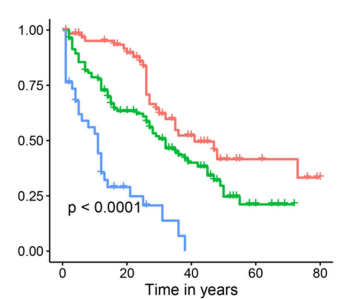

Number at risk

Figure 7 Subgroup analyses of nomograms. The survival curves show the distinct OS in age $<80$ (A), age $\geq 80$ (A), grade I-II (B), and grade III-IV (B) subgroups in the training cohort; The survival curves to show the distinct CSS in age $<80$ (C), age $\geq 80$ (C), grade I-II (D), and grade III-IV (D) subgroups in the training cohort; The survival curves to show the distinct OS in age $<80(\mathbf{E})$, age $\geq 80(\mathbf{E})$, grade I-II $(\mathbf{F})$, and grade III-IV $(\mathbf{F})$ subgroups in the validation cohort; The survival curves to show the distinct CSS in age $<80(\mathbf{G})$, age $\geq 80(\mathbf{G})$, grade I-II $(\mathbf{H})$, and grade III-IV $(\mathbf{H})$ subgroups in the validation cohort.

Abbreviations: OS, overall survival; CSS, cancer-specific survival. 

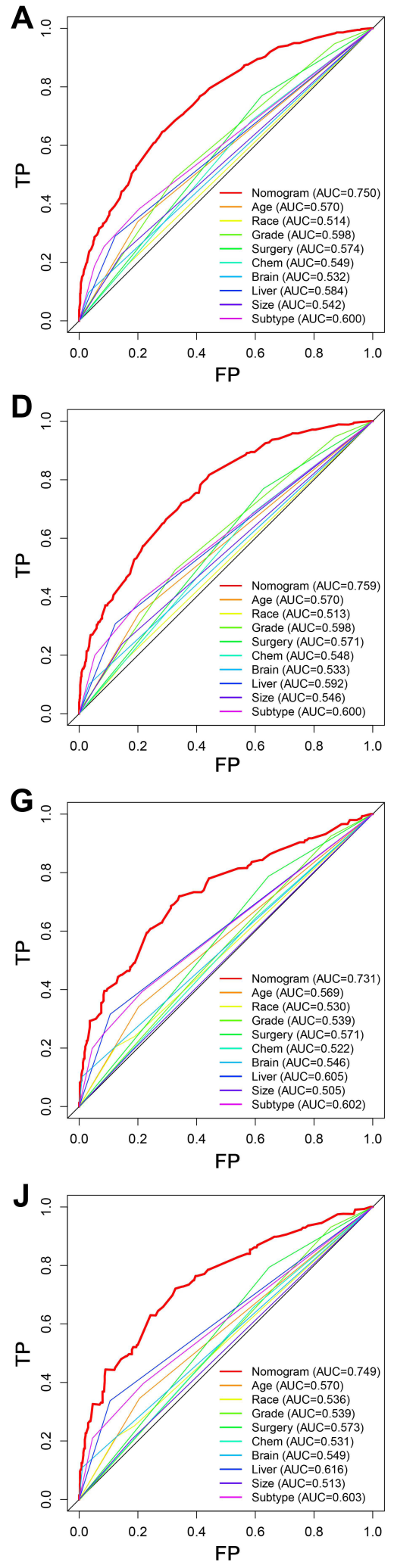

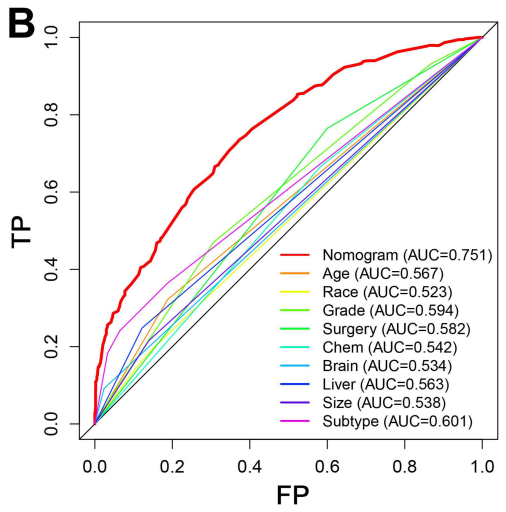

$\mathbf{E}_{\text {。 }}$
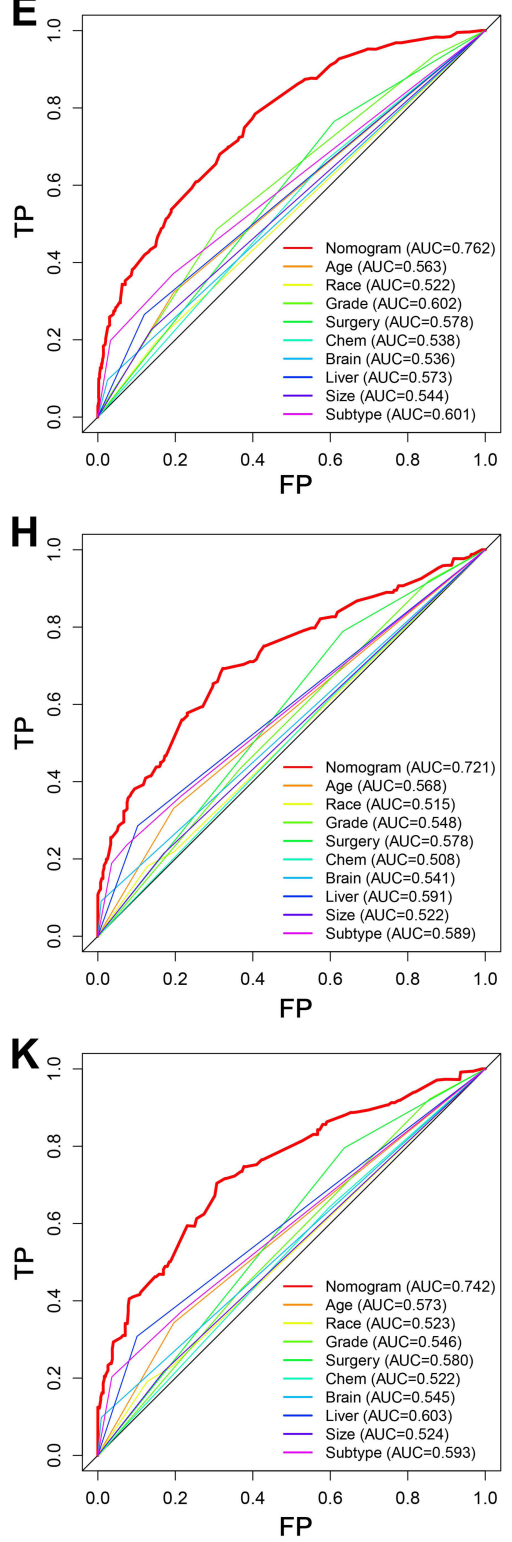
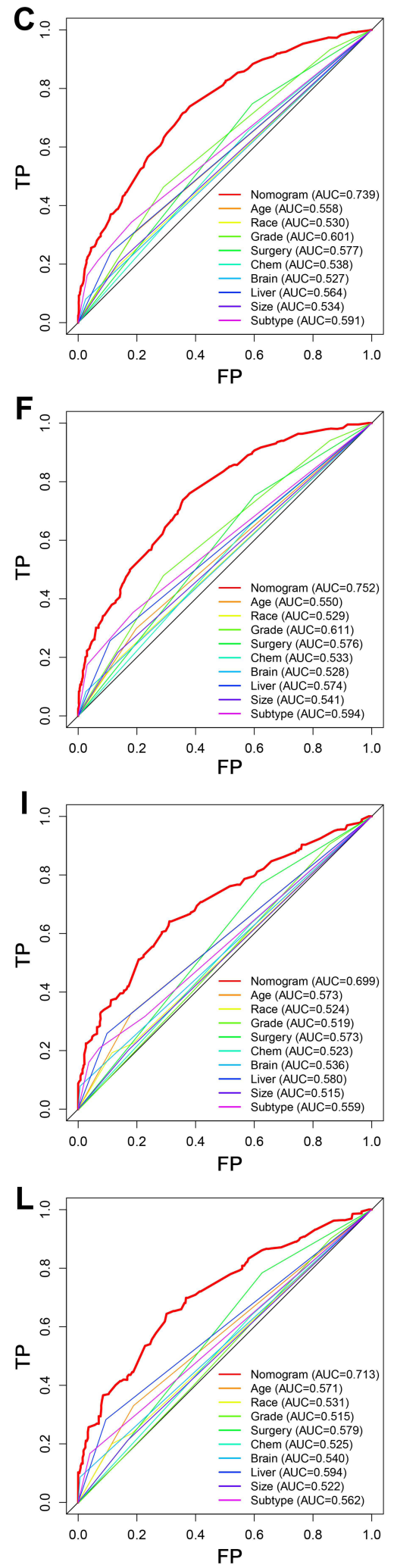

Figure 8 Comparison of AUC values between nomogram and single independent factors. The ROC curves of OS nomogram and all independent predictors at I2 (A), 18 (B), and 24 months (C) in the training cohort; The ROC curves of CSS nomogram and all independent predictors at 12 (D), 18 (E), and 24 months (F) in the training cohort; The ROC curves of OS nomogram and all independent predictors at I2 (G), 18 (H), and 24 months (I) in the validation cohort; The ROC curves of CSS nomogram and all independent predictors at $12(\mathbf{J}), 18(\mathbf{K})$, and 24 months $(\mathbf{L})$ in the validation cohort.

Abbreviations: TP, true positive; FP, false positive; AUC, area under the curve; ROC, receiver operating characteristic; OS, overall survival; CSS, cancer-specific survival. 


\section{Abbreviations}

$\mathrm{BC}$, breast cancer; OS, overall survival; CSS, cancerspecific survival; BCBM, breast cancer patients with bone metastasis; AUC, area under the curve; DCA, decision curve analysis; BM, bone metastasis; SEER, Surveillance, Epidemiology, and End Results; K-M, Kaplan-Meier; SD, standard deviation; IQR, interquartile ranges; OR, odds ratio; $\mathrm{HR}$, hazard ratio.

\section{Ethics Approval and Informed Consent}

The study was conducted in accordance with the Declaration of Helsinki (as revised in 2013). All information from the SEER program is available and free for public and approval was obtained from the Academic Ethics Committee of Shaoxing People's Hospital.

\section{Funding}

There is no funding to report.

\section{Disclosure}

The authors report no conflicts of interest in this work.

\section{References}

1. Siegel RL, Miller KD, Jemal A. Cancer statistics, 2019. CA Cancer J Clin. 2019;69(1):7-34. doi:10.3322/caac.21551

2. Graham N, Qian B-Z. Mesenchymal stromal cells: emerging roles in bone metastasis. Int J Mol Sci. 2018;19(4):1121. doi:10.3390/ ijms 19041121

3. Wang Z, Wang H, Ding X, Chen X, Shen K. A large-cohort retrospective study of metastatic patterns and prognostic outcomes between inflammatory and non-inflammatory breast cancer. Ther Adv Med Oncol. 2020;12:175883592093267. doi:10.1177/1758835920932674

4. Wang Z, Wang H, Sun X, Fang Y, Shen KW. A risk stratification model for predicting overall survival and surgical benefit in triple-negative breast cancer patients with de novo distant metastasis. Front Oncol. 2020;10:14. doi:10.3389/fonc.2020.00014

5. Nakai Y, Okamoto K, Terashima A, et al. Efficacy of an orally active small-molecule inhibitor of RANKL in bone metastasis. Bone Res. 2019;7(1):1-10. doi:10.1038/s41413-018-0036-5

6. Yao Y-B, Zheng X-E, Luo X-B, Wu A-M. Incidence, prognosis and nomograms of breast cancer with bone metastases at initial diagnosis: a large population-based study. Am J Transl Res. 2021;13 (9):10248-10261.

7. Svensson E, Christiansen CF, Ulrichsen SP, Rørth MR, Sørensen HT. Survival after bone metastasis by primary cancer type: a Danish population-based cohort study. BMJ Open. 2017;7(9):e016022. doi:10.1136/bmjopen-2017-016022

8. Park S, Yoon J-K, Lee SJ, Kang SY, Yim H, An Y-S. Prognostic utility of FDG PET/CT and bone scintigraphy in breast cancer patients with bone-only metastasis. Medicine. 2017;96(50):e8985.

9. Shizuku M, Shibata M, Okumura M, Takeuchi D, Kikumori T, Mizuno Y. Utility of urinary type I collagen cross-linked $\mathrm{N}$-telopeptide as a prognostic indicator in breast cancer patients with bone metastases. Breast Cancer. 2020;27(6):1065-1071. doi:10.1007/ s12282-020-01109-9
10. Parkes A, Warneke CL, Clifton K, et al. Prognostic factors in patients with metastatic breast cancer with bone-only metastases. Oncologist. 2018;23(11):1282. doi:10.1634/theoncologist.2018-0085

11. Luo A, Wu F, Han R, et al. Clinicopathological features and prognostic evaluation of bone metastasis in triple-negative breast cancer. J Cancer Res Ther. 2017;13(5):778. doi:10.4103/jcrt.JCRT 54317

12. Ferreira A, Alho I, Vendrell I, et al. The prognostic role of RANK SNP rs34945627 in breast cancer patients with bone metastases. Oncotarget. 2016;7(27):41380. doi:10.18632/oncotarget.9356

13. Foerster R, Bruckner T, Bostel T, Schlampp I, Debus J, Rief H. Prognostic factors for survival of women with unstable spinal bone metastases from breast cancer. Radiat Oncol. 2015;10(1):1-4. doi:10.1186/s13014-015-0458-9

14. Liu S, Song A, Zhou X, et al. ceRNA network development and tumour-infiltrating immune cell analysis of metastatic breast cancer to bone. J Bone Oncol. 2020;24:100304. doi:10.1016/j.jbo.2020.100304

15. Zhao C, Lou Y, Wang Y, et al. A gene expression signature-based nomogram model in prediction of breast cancer bone metastases. Cancer Med. 2019;8(1):200-208. doi:10.1002/cam4.1932

16. Wang Z, Cheng Y, Chen S, et al. Novel prognostic nomograms for female patients with breast cancer and bone metastasis at presentation. Ann Transl Med. 2020;8(5):197. doi:10.21037/ atm.2020.01.37

17. Deng J, Zhang D, Zhang W, Li J. Construction and validation of new nomograms to predict risk and prognostic factors of breast cancer bone metastasis in asian females: a Population-Based Retrospective Study. Int J Gen Med. 2021;14:8881-8902. doi:10.2147/IJGM.S335123

18. Lyu X, Luo B. Prognostic factors and survival prediction in HER2-positive breast cancer with bone metastases: a retrospective cohort study. Cancer Med. 2021;10(22):8114-8126. doi:10.1002/ cam 4.4326

19. Tu Q, Hu C, Zhang H, et al. Establishment and validation of novel clinical prognosis nomograms for luminal a breast cancer patients with bone metastasis. Biomed Res Int. 2020;2020:1972064. doi: $10.1155 / 2020 / 1972064$

20. Ma D, Jiang YZ, Xiao Y, et al. Integrated molecular profiling of young and elderly patients with triple-negative breast cancer indicates different biological bases and clinical management strategies. Cancer. 2020;126(14):3209-3218. doi:10.1002/cncr.32922

21. Nikolaidi A, Kotoula V, Koliou G-A, et al. Tumor mutational patterns and infiltrating lymphocyte density in young and elderly patients with breast cancer. Cancer Genom Proteom. 2020;17(2):181-193. doi:10.21873/cgp.20179

22. Johansson $\mathrm{AL}$, Trewin $\mathrm{CB}$, Hjerkind $\mathrm{KV}$, Ellingjord-Dale $\mathrm{M}$, Johannesen TB, Ursin G. Breast cancer-specific survival by clinical subtype after 7 years follow-up of young and elderly women in a nationwide cohort. Int $J$ Cancer. 2019;144(6):1251-1261. doi:10.1002/ijc. 31950

23. Martinez-Ramos D, Simon-Monterde L, Queralt-Martin R, SuelvesPiqueres C, Menor-Duran P, Escrig-Sos J. Breast cancer in octogenarian. Are we doing our best? A population-registry based study. Breast. 2018;38:81-85. doi:10.1016/j.breast.2017.12.007

24. Chu J, Yang D, Wang L, Xia J. Nomograms predicting survival for all four subtypes of breast cancer: a SEER-based population study. Ann Transl Med. 2020;8(8):544. doi:10.21037/atm-20-2808

25. Camp RL, Dolled-Filhart M, Rimm DL. X-tile: a new bio-informatics tool for biomarker assessment and outcome-based cut-point optimization. Clin Cancer Res. 2004;10(21):7252-7259. doi:10.1158/1078-0432.CCR-04-0713

26. Bertolo A, Rosso C, Voutsadakis IA. Breast cancer in patients 80 years-old and older. Eur $J$ Breast Health. 2020;16(3):208. doi:10.5152/ejbh.2020.5659

27. La Rocca E, Meneghini E, Lozza L, et al. Older age and comorbidity in breast cancer: is RT alone the new therapeutic frontier? $J$ Cancer Res Clin Oncol. 2020;146(7):1791-1800. doi:10.1007/s00432-02003243-5 
28. Delgado-Ramos GM, Nasir SS, Wang J, Schwartzberg LS. Real-world evaluation of effectiveness and tolerance of chemotherapy for early-stage breast cancer in older women. Breast Cancer Res Treat. 2020;182(2):247-258. doi:10.1007/s10549-020-05684-5

29. Ji P, Gong Y, Jiang -C-C, Hu X, Di G-H, Shao Z-M. Association between socioeconomic factors at diagnosis and survival in breast cancer: a population-based study. Cancer Med. 2020;9 (5):1922-1936. doi:10.1002/cam4.2842

30. Huang Q, Xu T-Y, Wu Z-Y. Construction and validation of nomograms for predicting overall survival and cancer-specific survival in nonmetastatic inflammatory breast cancer patients receiving tri-modality therapy: a Population-Based Study. Med Sci Monit. 2019;25:9167-9178. doi:10.12659/MSM.919458

31. Diao J, Ma L, Sun M, et al. Construction and validation of a nomogram to predict overall survival in patients with inflammatory breast cancer. Cancer Med. 2019;8(12):5600-5608. doi:10.1002/cam4.2470

32. Liu D, Wu J, Lin C, et al. Breast subtypes and prognosis of breast cancer patients with initial bone metastasis: a Population-Based Study. Front Oncol. 2020;10:580112. doi:10.3389/fonc.2020.580112

33. Li X, Zhang X, Liu J, Shen Y. Prognostic factors and survival according to tumour subtype in women presenting with breast cancer bone metastases at initial diagnosis: a SEER-based study. $B M C$ Cancer. 2020;20(1):1102. doi:10.1186/s12885-020-07593-8

34. Chen S, Wan Q, Zhou D, et al. A simple-to-use nomogram for predicting the survival of early hepatocellular carcinoma patients. Front Oncol. 2019;9:584. doi:10.3389/fonc.2019.00584

35. Wo Y, Yang H, Zhang Y, Wo J. Development and external validation of a nomogram for predicting survival in patients with stage IA nonsmall cell lung cancer $\leq 2 \mathrm{~cm}$ undergoing sublobectomy. Front Oncol. 2019;9. doi:10.3389/fonc.2019.01385

36. Rhu J, Lee SK, Kil WH, Lee JE, Nam SJ. Surgery of primary tumour has survival benefit in metastatic breast cancer with single-organ metastasis, especially bone. ANZ J Surg. 2015;85(4):240-244. doi:10.1111/ans. 12548
37. Gu Y, Wu G, Zou X, Huang P, Yi L. Prognostic value of site-specific metastases and surgery in de novo stage IV triple-negative breast cancer: a population-based analysis. Med Sci Monit. 2020;26: e920432. doi:10.12659/MSM.920432

38. Botteri E, Munzone E, Intra M, et al. Role of breast surgery in T1-3 breast cancer patients with synchronous bone metastases. Breast Cancer Res Treat. 2013;138(1):303-310. doi:10.1007/s10549-013-2449-6

39. Xiong Z, Deng G, Wang J, et al. Could local surgery improve survival in de novo stage IV breast cancer? BMC Cancer. 2018;18 (1):885. doi:10.1186/s12885-018-4767-x

40. Xiong Z, Deng G, Huang X, et al. Bone metastasis pattern in initial metastatic breast cancer: a population-based study. Cancer Manag Res. 2018;10:287-295. doi:10.2147/CMAR.S155524

41. Turanli S. The role of surgery in breast cancer patients with isolated bone metastases at the time of diagnosis. J Surg Oncol. 2009;100 (2):95-99. doi:10.1002/jso.21300

42. Oruç Z, Kaplan MA, Arslan Ç. An update on the currently available and future chemotherapy for treating bone metastases in breast cancer patients. Expert Opin Pharmacother. 2018;19(12):1305-1316. doi:10.1080/14656566.2018.1504922

43. Geels P, Eisenhauer E, Bezjak A, Zee B, Day A. Palliative effect of chemotherapy: objective tumor response is associated with symptom improvement in patients with metastatic breast cancer. J Clin Oncol. 2000;18(12):2395-2405. doi:10.1200/JCO.2000.18.12.2395

44. Zheng Y, Zhong G, Yu K, Lei K, Yang Q. Individualized prediction of survival benefit from locoregional surgical treatment for patients with metastatic breast cancer. Front Oncol. 2020;10:148. doi: $10.3389 /$ fonc. 2020.00148
International Journal of General Medicine

\section{Publish your work in this journal}

The International Journal of General Medicine is an international, peer-reviewed open-access journal that focuses on general and internal medicine, pathogenesis, epidemiology, diagnosis, monitoring and treatment protocols. The journal is characterized by the rapid reporting of reviews, original research and clinical studies

\section{Dovepress}

across all disease areas. The manuscript management system is completely online and includes a very quick and fair peer-review system, which is all easy to use. Visit http://www.dovepress.com/ testimonials.php to read real quotes from published authors. 